\title{
Chronic Nephrotoxic Nephritis in Spontaneously Hypertensive Rats
}

\author{
Kenzo Motoki, Yoshio Kusuyama, Yuji Ueno, \\ Yasumasa MiYamoto, Ichiro Nishio, \\ and Yoshiaki Masurama
}

The role of hypertension in chronic glomerulonephritis remains unsolved. From the glomerular changes until 6 weeks after the injection of nephrotoxic serum (NTS) in Wistar rats (WR) and spontaneously hypertensive rats (SHR), our previous results suggested that sustained hypertension might aggravate the nephrotoxic processes or protract the course of glomerulonephritis. The present study was attemped to observe the more prolonged glomerular changes of nephrotoxic nephritis (NTN) until 15 weeks after the injection of NTS in SHR, compared with control WR.

Materials and Methods:

NTS was prepared by the method of Gang et al. A dose of $1.0 \mathrm{ml}$ of NTS was injected into the tail veins in the male 13 SHR and $9 \mathrm{WR}$, weighing 100-110 $\mathrm{Gm}$. One $\mathrm{ml}$ of physiological saline was similarly injected in the male 17 SHR and $9 \mathrm{WR}$ as controls. Blood pressure was measured once a week by the tail plethysmographic technique in unanesthetized condition after warming at $45^{\circ} \mathrm{C}$ for $4 \mathrm{~min}$. Urinary protein was measured semiquantitatively by Albstix (Ames Co). These rats were sacrificed 15 weeks after the injection, and the kidney specimens were examined by the light microscopic and the immunofluonescent technique using FITC labeled anti-rat IgG rabbit serum and FITC labeled anti-fibrinogen bovine serum. The blood samples were taken by cutting the tail tip. Plasma renin activity (PRA) and plasma aldosterone concentration (PAG) were measured by the radioimmunoassay in the control period and, 8 and 14 weeks after the injection of NTS. The remaining ratio of ${ }^{131} \mathrm{I}-$ Hippuran in blood was calculated as an index of renal function, and circulating blood volume (BV) was measured by ${ }^{125}$ I-RISA dilution technique.

Results :

More than $1000 \mathrm{mg} / 100 \mathrm{ml}$ of urine protein appeared 2 to 3 days after NTS injection and proteinuria continued for several weeks. In 5 weeks after the injection of NTS, proteinuria in WR was gradually decreased, while it remained constant even for 14 weeks in SHR. Fifteen weeks after injection, only mild mesangial proliferation and scattered adhesion of capillary tuft were observed in glomeruli of WR, while moderately increased mesangial matrix, localized hyalinization in glomeruli and marked crescent formation were shown in SHR.

The remaining ratio of ${ }^{131} \mathrm{I}$-Hippuran in the blood in $20 \mathrm{~min}$ was $11.0 \pm 0.15 \%$

From the Division of Cardiology, Department of Medicine, Wakayama Medical College, Wakayama. 
in control of $\mathrm{WR}$ and $11.0 \pm 0.16 \%$ in NTN group of WR, while the ratio in SHR was $8.3 \pm 0.3 \%$ in control and $24.7 \pm 2.7 \%$ in NTN group. Significant difference was shown between NTN groups of SHR and WR $(P<0.001)$. Thus, the kidney function was more severely damaged in this phase of NTN in SHR than WR, corresponding to the histological changes.

PRA was $4.10 \pm 0.18 \mathrm{ng} / 100 \mathrm{ml} / \mathrm{hr}$ in control periods before the injection in WR. PRA 8 weeks after the injection in WR was $2.93 \pm 0.16$ in NTN group and $3.40 \pm 0.22$ in the control. Fourteen weeks after the injection the values were 3.41 \pm 0.14 and $3.87 \pm 0.28$, respectively. PRA in SHR was $5.00 \pm 0.18 \mathrm{ng} / \mathrm{ml} / \mathrm{hr}$ before the injection. PRA in 8 weeks was $4.09 \pm 0.40$ in NTN group and $4.73 \pm 0.17$ in the control of SHR. In 14 weeks the values in SHR were $3.15 \pm 0.04$ and $5.12 \pm$ 0.15 , respectively. PRA was significantly suppressed in NTS injected SHR compared with the control of SHR in 14 weeks $(\mathrm{P}<0.001)$.

PAC was $37.6 \pm 3.1 \mathrm{ng} / 100 \mathrm{ml}$ in NTN group of $\mathrm{WR}$ and $42.3 \pm 4.2$ in the control in 8 weeks. PAC in 14 weeks was $30.9 \pm 2.1$ in NTN group and $44.0 \pm 1.2$ in the control. PAC was $66.5 \pm 11.4 \mathrm{ng} / 100 \mathrm{ml}$ in NTN group of SHR and $82.7 \pm 8.4$ in the control in 8 weeks. PAC in 14 weeks in SHR the values were $43.5 \pm 2.5$ and $87.8 \pm 8.8$, respectively. PAC was also significantly suppressed in NTS injected group in 14 weeks $(P<0.001)$. BV in NTN group $(6.87 \pm 0.16 \mathrm{ml} / 100 \mathrm{Gm}$ of body weight) was significantly increased as compared with the control $(6.20 \pm 0.11)$ in WR $(\mathrm{P}<0.01)$. BV in NTN group $(7.62 \pm 0.18 \mathrm{ml} / 100 \mathrm{Gm} \mathrm{B.W.})$ was also increased in SHR as compared with the control $(6.96 \pm 0.13)(\mathrm{P}<0.02)$. There were no significant differences in blood pressure courses between NTN and control groups both in SHR and WR.

\section{Discussion:}

The effect of hypertension on chronic glomerulonephritis was observed in SHR combined with nephrotoxic nephritis.

The histological glomerular changes still continued in 14 weeks in SHR, though the changes diminished in control WR. There was no significant difference in the blood pressure course between NTN and the control groups of SHR. Proteinuria remained positive in NTN group of SHR, though it diminished in the group of WR. Circulating blood volume was increased and the renal function, measured by the remaining ratio of ${ }^{131}$ I-Hippuran in blood was more reduced in NTN group of SHR than that of WR. Plasma renin activity and plasma aldosterone were suppressed in NTN group than the controls.

Thus, the histological renal changes were more marked in SHR in this phase of nephrotoxic nephritis than in WR, and the renal function was more diminished and plasma volume was more increased in NTN groups of SHR than the control.

Conclusion:

1) Histological glomerular changes were more marked in 15 weeks after nephrotoxic serum injection in SHR than in WR.

2) No significant difference was found in the blood pressure course between the nephrotoxic nephritis group and the control in SHR.

3) Plasma renin activity and plasma aldosterone were suppressed, circulating blood volume was in creased and the renal function was diminished in neph- 
rotoxic nephritis group of SHR, compared with the controls.

Therefore, it is concluded that hypertension might protract the nephritic process and rather aggravate the course of chronic glomerulonephritis. 